\title{
Cauchy integral formulae in quaternionic Hermitean Clifford analysis
}

\author{
Ricardo Abreu-Blaya Juan Bory-Reyes Fred Brackx \\ Hennie De Schepper Frank Sommen
}

\begin{abstract}
The theory of complex Hermitean Clifford analysis was developed recently as a refinement of Euclidean Clifford analysis; it focusses on the simultaneous null solutions, called Hermitean monogenic functions, of two Hermitean Dirac operators constituting a splitting of the traditional Dirac operator. In this function theory, the fundamental integral representation formulae, such as the Borel-Pompeiu and the Clifford-Cauchy formula have been obtained by using a $(2 \times 2)$ circulant matrix formulation. In the meantime, the basic setting has been established for so-called quaternionic Hermitean Clifford analysis, a theory centered around the simultaneous null solutions, called q-Hermitean monogenic functions, of four Hermitean Dirac operators in a quaternionic Clifford algebra setting. In this paper we address the problem of establishing a quaternionic Hermitean Clifford-Cauchy integral formula, by following a $(4 \times 4)$ circulant matrix approach.
\end{abstract}

Keywords. quaternionic Hermitean Clifford analysis, Cauchy integral formula. Mathematics Subject Classification (2000). 30G35.

\section{Introduction}

There exist two major ways in which the Cauchy integral formula for holomorphic functions in the complex plane can be generalized to the case of several complex variables: one may consider a holomorphic kernel and an integral over the distinguished boundary $\partial_{0} \widetilde{D}$ of a polydisk $\widetilde{D}=\prod_{j=1}^{n} \widetilde{D}_{j}$ in $\mathbb{C}^{n}$, resulting into

$$
f\left(z_{1}, \ldots, z_{n}\right)=\frac{1}{(2 \pi i)^{n}} \int_{\partial_{0} \widetilde{D}} \frac{f\left(\xi_{1}, \ldots, \xi_{n}\right)}{\left(\xi_{1}-z_{1}\right) \cdots\left(\xi_{n}-z_{n}\right)} d \xi_{1} \wedge \cdots \wedge d \xi_{n}, z_{j} \in \stackrel{\circ}{D}_{j},
$$

or one may take an integral over the smooth boundary $\partial D$ of a bounded domain $D$ in $\mathbb{C}^{n}$, in combination with a kernel which is no longer holomorphic but still 
harmonic, resulting into the Martinelli-Bochner formula, see e.g. [16], which reads:

$$
f(z)=\int_{\partial D} f(\xi) \frac{(n-1) !}{(2 \pi i)^{n}} \sum_{j=1}^{n}(-1)^{j-1} \frac{\xi_{j}^{c}-z_{j}^{c}}{|\xi-z|^{2 n}} \widetilde{\widehat{d \xi_{j}^{c}}}, \quad z \in \stackrel{\circ}{D}
$$

where.$^{c}$ denotes complex conjugation and $\widetilde{\widetilde{d \xi_{j}^{c}}}=d \xi_{1}^{c} \wedge \cdots \wedge d \xi_{j-1}^{c} \wedge d \xi_{j+1}^{c} \wedge \cdots \wedge$ $d \xi_{n}^{c} \wedge d \xi_{1} \wedge \cdots \wedge d \xi_{n}$ is an oriented surface element. For detailed information on this formula, which reduces to the traditional Cauchy integral formula when $n=1$, we refer the reader to e.g. [15].

Alternatively, the Cauchy integral formula was generalized within the framework of Clifford analysis, where functions are considered defined on Euclidean space $\mathbb{R}^{m}$, taking their values in a Clifford algebra and which moreover are null solutions of a first order vector valued differential operator, called Dirac operator, which factorizes the Laplacian. Such functions are called monogenic: they are a generalization of holomorphic functions in the complex plane and, at the same time, a refinement of harmonic functions. The Dirac operator being rotation invariant, the name Euclidean Clifford analysis is used to refer to this setting. Standard references are $[4,14,11,13,12]$. The kernel appearing in the CliffordCauchy formula is monogenic, up to a pointwise singularity, while the integral is taken over the complete boundary. For a function $f$ monogenic in the open region $\Omega$ and a bounded domain $D$ with smooth boundary $\partial D$, such that $\bar{D} \subset \Omega$, one has

$$
f(\underline{X})=\frac{1}{a_{m}} \int_{\partial D} \frac{\overline{\bar{\xi}}-\underline{\bar{X}}}{|\underline{\xi}-\underline{X}|^{m}} d \sigma_{\underline{\xi}} f(\underline{\xi}), \quad \underline{X} \in \stackrel{\circ}{D}
$$

with $a_{m}$ the area of the unit sphere $S^{m-1}$ in $\mathbb{R}^{m}$, $\cdot$ the Clifford conjugation and $d \sigma_{\xi}$ a Clifford algebra valued differential form of order $m-1$. This formula has been a corner stone in the development of the monogenic function theory.

More recently Hermitean Clifford analysis has emerged as yet a refinement of the Euclidean setting, for the case of $\mathbb{R}^{2 n} \cong \mathbb{C}^{n}$. Here, Hermitean monogenic functions are considered. These are functions taking values either in the complex Clifford algebra $\mathbb{C}_{2 n}$ or in complex spinor space $\mathbb{S}$, and being simultaneous null solutions of two complex Hermitean Dirac operators which are invariant under the action of the unitary group. The study of complexified Dirac operators, also in other settings, was initiated in $[19,18,20]$; the systematic development of the Hermitean function theory was continued in e.g. $[5,6,7]$. In $[8,2,1]$ a Cauchy integral formula for Hermitean monogenic functions was established by means of a matrix formulation with circulant $(2 \times 2)$ matrix functions, in domains with respectively smooth and fractal boundaries, see also [3]. Observe that the Cauchy integral formula in this setting indeed was expected to differ from the traditional form above, since in some particular cases Hermitean monogenicity is equivalent with holomorphy in the underlying complex variables, see [6]. 
In the recent papers [17],[10],[9] the Hermitean Clifford setting was further generalized by considering functions with values in a quaternionic Clifford algebra. The authors in [17] introduce the quaternionic Hermitean Dirac equation, which corresponds to functions being simultaneous null solutions of four mutually related quaternionic Dirac operators. In this contribution we establish a Clifford-Cauchy integral formula within this new function theory, for which the key idea is the introduction of a $(4 \times 4)$ circulant matrix setting, which is shown to be the correct way of handling the operators and functions involved. As an application, we show that, when considering a special case, our Clifford-Cauchy formula reduces to the Martinelli-Bochner type formula introduced in [17].

\section{Preliminaries}

Let $\left(e_{1}, \ldots, e_{m}\right)$ be an orthonormal basis of Euclidean space $\mathbb{R}^{m}$ and let $\mathbb{R}_{0, m}$ be the real Clifford algebra of signature $(0, m)$ constructed over $\mathbb{R}^{m}$. The noncommutative multiplication in $\mathbb{R}_{0, m}$ is governed by the rules $e_{j} e_{k}+e_{k} e_{j}=-2 \delta_{j k}$, $j, k=1, \ldots, m$. In $\mathbb{R}_{0, m}$ one can consider the following automorphisms:

(i) the conjugation, given by $\bar{e}_{r}=-e_{r}$ and $\overline{a b}=\bar{b} \bar{a}$ for any $a$ and $b$ in $\mathbb{R}_{0, m}$;

(ii) the main involution, given by $\widetilde{e}_{r}=-e_{r}$ and $\widetilde{a b}=\widetilde{a} \widetilde{b}$ for any $a$ and $b$ in $\mathbb{R}_{0, m}$.

Consider then the skew-field of quaternions $\mathbb{H}$ with elements $q=x_{0}+i x_{1}+j x_{2}+k x_{3}$, where $i^{2}=j^{2}=k^{2}=-1$ and $i j=-j i=k$. The algebra of quaternions may be identified with the Clifford algebra $\mathbb{R}_{0,2}$ making the identifications $i \leftrightarrow e_{1}$, $j \leftrightarrow e_{2}$ and $k \leftrightarrow e_{1} e_{2}$. The above automorphisms (i)-(ii) respectively lead to the $\mathbb{H}-$-conjugation

$$
\bar{q}=x_{0}-i x_{1}-j x_{2}-k x_{3}
$$

and to the main $\mathbb{H}$-involution

$$
q^{\gamma} \equiv \tilde{q}=x_{0}-i x_{1}-j x_{2}+k x_{3} .
$$

However, it is quite natural to introduce two similar $\mathbb{H}-$-involutions defined by

$$
\begin{aligned}
q^{\alpha} & =x_{0}+i x_{1}-j x_{2}-k x_{3}, \\
q^{\beta} & =x_{0}-i x_{1}+j x_{2}-k x_{3} .
\end{aligned}
$$

Now we consider the quaternionic Clifford algebra $\mathbb{H}_{m}=\mathbb{H} \otimes_{\mathbb{R}} \mathbb{R}_{0, m}$, with elements $\lambda=\sum_{A} e_{A} \lambda_{A}, \lambda_{A} \in \mathbb{H}$, where $e_{A}$ are the basis elements of $\mathbb{R}_{0, m}$ generated by $\left(e_{1}, \ldots, e_{m}\right)$ and their multiplication rules above, and where it is understood that the quaternionic scalars commute with the Clifford basis vectors. The quaternionic Hermitean conjugate of $\lambda \in \mathbb{H}_{m}$ is defined as the composition of the $\mathbb{H}$-conjugation and the Clifford conjugation in $\mathbb{R}_{0, m}$, i.e. $\lambda^{\dagger}=\sum_{A} \overline{e_{A}} \overline{\lambda_{A}}$. We also define a norm in $\mathbb{H}_{m}:|\lambda|=\left[\lambda \lambda^{\dagger}\right]_{0}=\sum_{A}\left|\lambda_{A}\right|^{2}$, where $[\cdot]_{0}$ stands for taking the scalar part.

From now on we assume the dimension to be of the form: $m=4 n$. 
Definition 1 ([17]). The quaternionic Witt basis of $\mathbb{H}_{m}=\mathbb{H} \otimes_{\mathbb{R}} \mathbb{R}_{m}$, with $m=4 n$, is given by $\left\{f_{l}, f_{l}^{\alpha}, f_{l}^{\beta}, f_{l}^{\gamma}\right\}, l=1, \ldots, n$, where

$$
\begin{aligned}
& f_{l}=e_{1+4(l-1)}-i e_{2+4(l-1)}-j e_{3+4(l-1)}-k e_{4+4(l-1)}, \\
& f_{l}^{\alpha}=e_{1+4(l-1)}-i e_{2+4(l-1)}+j e_{3+4(l-1)}+k e_{4+4(l-1)}, \\
& f_{l}^{\beta}=e_{1+4(l-1)}+i e_{2+4(l-1)}-j e_{3+4(l-1)}+k e_{4+4(l-1)}, \\
& f_{l}^{\gamma}=e_{1+4(l-1)}+i e_{2+4(l-1)}+j e_{3+4(l-1)}-k e_{4+4(l-1)} .
\end{aligned}
$$

Let us now define, see [17], the following real Clifford vectors associated to an element $\left(x_{1}, \ldots, x_{4 n}\right)$ in $\mathbb{R}^{4 n}$ :

$$
\begin{aligned}
\underline{X}=\underline{X}_{0} & =\sum_{l=1}^{n}\left(e_{4 l-3} x_{4 l-3}+e_{4 l-2} x_{4 l-2}+e_{4 l-1} x_{4 l-1}+e_{4 l} x_{4 l}\right), \\
\underline{X}_{1} & =\sum_{l=1}^{n}\left(e_{4 l-3} x_{4 l-2}-e_{4 l-2} x_{4 l-3}-e_{4 l-1} x_{4 l}+e_{4 l} x_{4 l-1}\right), \\
\underline{X}_{2} & =\sum_{l=1}^{n}\left(e_{4 l-3} x_{4 l-1}+e_{4 l-2} x_{4 l}-e_{4 l-1} x_{4 l-3}-e_{4 l} x_{4 l-2}\right), \\
\underline{X}_{3} & =\sum_{l=1}^{n}\left(e_{4 l-3} x_{4 l}-e_{4 l-2} x_{4 l-1}+e_{4 l-1} x_{4 l-2}-e_{4 l} x_{4 l-3}\right) .
\end{aligned}
$$

Note that $\underline{X}_{0}$ indeed corresponds to the usual Clifford vector, while $\underline{X}_{1}, \underline{X}_{2}, \underline{X}_{3}$ are similar to the twisted vector $\underline{X} \mid$ introduced in the complex Hermitean case, see $[7,5]$. An easy computation shows that

$$
\begin{gathered}
\underline{X}_{0}^{2}=\underline{X}_{1}^{2}=\underline{X}_{2}^{2}=\underline{X}_{3}^{2}=-|\underline{X}|^{2}, \\
\left\{\underline{X}_{r}, \underline{X}_{s}\right\} \equiv \underline{X}_{r} \underline{X}_{s}+\underline{X}_{s} \underline{X}_{r}=0, \quad r, s=0,1,2,3, r \neq s .
\end{gathered}
$$

Next one defines the differential operators

$$
\begin{aligned}
\partial_{\underline{X}}=\partial_{\underline{X}_{0}} & =\sum_{l=1}^{n}\left(e_{4 l-3} \partial_{x_{4 l-3}}+e_{4 l-2} \partial_{x_{4 l-2}}+e_{4 l-1} \partial_{x_{4 l-1}}+e_{4 l} \partial_{x_{4 l}}\right), \\
\partial_{\underline{X}_{1}} & =\sum_{l=1}^{n}\left(e_{4 l-3} \partial_{x_{4 l-2}}-e_{4 l-2} \partial_{x_{4 l-3}}-e_{4 l-1} \partial_{x_{4 l}}+e_{4 l} \partial_{x_{4 l-1}}\right), \\
\partial_{\underline{X}_{2}} & =\sum_{l=1}^{n}\left(e_{4 l-3} \partial_{x_{4 l-1}}+e_{4 l-2} \partial_{x_{4 l}}-e_{4 l-1} \partial_{x_{4 l-3}}-e_{4 l} \partial_{x_{4 l-2}}\right), \\
\partial_{\underline{X}_{3}} & =\sum_{l=1}^{n}\left(e_{4 l-3} \partial_{x_{4 l}}-e_{4 l-2} \partial_{x_{4 l-1}}+e_{4 l-1} \partial_{x_{4 l-2}}-e_{4 l} \partial_{x_{4 l-3}}\right),
\end{aligned}
$$


which are the Fischer (or Fourier) duals of the above vectors $\underline{X}_{0}, \underline{X}_{1}, \underline{X}_{2}, \underline{X}_{3}$. Again, note that $\partial_{\underline{X}_{0}}$ corresponds to the usual Dirac operator $\partial_{\underline{X}}$, while $\partial_{\underline{X}_{1}}, \partial_{\underline{X}_{2}}$, $\partial_{\underline{X}_{3}}$ are analogues of the twisted Dirac operator $\partial_{\underline{X}}$ in the complex Hermitean case. Similar to (1)-(2) we have the relations

$$
\begin{gathered}
\partial_{\underline{X}_{0}}^{2}=\partial_{\underline{X}_{1}}^{2}=\partial_{\underline{X}_{2}}^{2}=\partial_{\underline{X}_{3}}^{2}=-\Delta_{4 n}, \\
\left\{\partial_{\underline{X}_{r}}, \partial_{\underline{X}_{s}}\right\} \equiv \partial_{\underline{X}_{r}} \partial_{\underline{X}_{s}}+\partial_{\underline{X}_{s}} \partial_{\underline{X}_{r}}=0, \quad r, s=0,1,2,3, r \neq s .
\end{gathered}
$$

Starting from these Euclidean variables and operators, their quaternionic Hermitean counterparts are defined. First, the quaternionic Hermitean variables are

$$
\begin{aligned}
\underline{Z}=\underline{Z}_{0} & =\underline{X}_{0}+i \underline{X}_{1}+j \underline{X}_{2}+k \underline{X}_{3}=\sum_{l=1}^{n} f_{l}\left(x_{4 l-3}+i x_{4 l-2}+j x_{4 l-1}+k x_{4 l}\right), \\
\underline{Z}_{1} & =\underline{X}_{0}+i \underline{X}_{1}-j \underline{X}_{2}-k \underline{X}_{3}=\sum_{l=1}^{n} f_{l}^{\alpha}\left(x_{4 l-3}+i x_{4 l-2}-j x_{4 l-1}-k x_{4 l}\right), \\
\underline{Z}_{2} & =\underline{X}_{0}-i \underline{X}_{1}+j \underline{X}_{2}-k \underline{X}_{3}=\sum_{l=1}^{n} f_{l}^{\beta}\left(x_{4 l-3}-i x_{4 l-2}+j x_{4 l-1}-k x_{4 l}\right), \\
\underline{Z}_{3} & =\underline{X}_{0}-i \underline{X}_{1}-j \underline{X}_{2}+k \underline{X}_{3}=\sum_{l=1}^{n} f_{l}^{\gamma}\left(x_{4 l-3}-i x_{4 l-2}-j x_{4 l-1}+k x_{4 l}\right) .
\end{aligned}
$$

Note that $\underline{Z}_{0} \underline{Z}_{0}^{\dagger}+\underline{Z}_{1} \underline{Z}_{1}^{\dagger}+\underline{Z}_{2} \underline{Z}_{2}^{\dagger}+\underline{Z}_{3} \underline{Z}_{3}^{\dagger}=\underline{Z}_{0}^{\dagger} \underline{Z}_{0}+\underline{Z}_{1}^{\dagger} \underline{Z}_{1}+\underline{Z}_{2}^{\dagger} \underline{Z}_{2}+\underline{Z}_{3}^{\dagger} \underline{Z}_{3}=16|\underline{X}|^{2}$.

The Hermitean Dirac operators are similarly derived from the Euclidean ones:

$$
\begin{aligned}
& \partial_{\underline{Z}_{0}}=\frac{1}{16}\left(\partial_{\underline{X}_{0}}+i \partial_{\underline{X}_{1}}+j \partial_{\underline{X}_{2}}+k \partial_{\underline{X}_{3}}\right), \\
& \partial_{\underline{Z}_{1}}=\frac{1}{16}\left(\partial_{\underline{X}_{0}}+i \partial_{\underline{X}_{1}}-j \partial_{\underline{X}_{2}}-k \partial_{\underline{X}_{3}}\right), \\
& \partial_{\underline{Z}_{2}}=\frac{1}{16}\left(\partial_{\underline{X}_{0}}-i \partial_{\underline{X}_{1}}+j \partial_{\underline{X}_{2}}-k \partial_{\underline{X}_{3}}\right), \\
& \partial_{\underline{Z}_{3}}=\frac{1}{16}\left(\partial_{\underline{X}_{0}}-i \partial_{\underline{X}_{1}}-j \partial_{\underline{X}_{2}}+k \partial_{\underline{X}_{3}}\right) .
\end{aligned}
$$

and may also be expressed in terms of the Witt basis. They establish the following decomposition of the Laplacian in $\mathbb{R}^{4 n}: \Delta_{4 n}=16\left(\partial_{\underline{Z}_{0}} \partial_{\underline{Z}_{0}}^{\dagger}+\partial_{\underline{Z}_{1}} \partial_{\underline{Z}_{1}}^{\dagger}+\partial_{\underline{Z}_{2}} \partial_{\underline{Z}_{2}}^{\dagger}+\right.$ $\left.\partial_{\underline{Z}_{3}} \partial_{\underline{Z}_{3}}^{\dagger}\right)=16\left(\partial_{\underline{Z}_{0}}^{\dagger} \partial_{\underline{Z}_{0}}+\partial_{\underline{Z}_{1}}^{\dagger} \partial_{\underline{Z}_{1}}+\partial_{\underline{Z}_{2}}^{\dagger} \partial_{\underline{Z}_{2}}+\partial_{\underline{Z}_{3}}^{\dagger} \partial_{\underline{Z}_{3}}\right)$.

Finally we introduce the central concept in quaternionic Hermitean Clifford analysis, see [17].

Definition 2. Let $\Omega$ be an open set in $\mathbb{R}^{4 n}$. A continuously differentiable function $f: \Omega \mapsto \mathbb{H}_{4 n}$ is said to be q-Hermitean monogenic in $\Omega$ iff it satisfies the system $\partial_{\underline{Z}_{0}} f=\partial_{\underline{Z}_{1}} f=\partial_{\underline{Z}_{2}} f=\partial_{\underline{Z}_{3}} f=0$, or, equivalently, the system $\partial_{\underline{X}_{0}} f=\partial_{\underline{X}_{1}} f=$ $\partial_{\underline{X}_{2}} f=\partial_{\underline{X}_{3}} f=0$.

Note that a $q$-Hermitean monogenic function in $\Omega$ is automatically monogenic in $\Omega$ and thus harmonic in $\Omega$. 


\section{A matrix approach}

In complex Hermitean Clifford analysis, a matrix approach has shown to be the key for establishing a Cauchy integral representation formula for Hermitean monogenic functions, see e.g. $[8,2]$. Seen the formal similarities between the complex and the quaternionic Hermitean Clifford setting, we will follow a similar strategy.

The fundamental solutions of the Dirac operators $\partial_{\underline{X}_{r}}, r=0,1,2,3$, i.e. the Euclidean Cauchy kernels, are respectively given by

$$
E_{r}(\underline{X})=-\frac{1}{a_{4 n}} \frac{\underline{X}_{r}}{|\underline{X}|^{4 n}}, \quad r=0,1,2,3
$$

where $a_{4 n}=\frac{2 \pi^{2 n}}{\Gamma(2 n)}$ denotes the area of the unit sphere $S^{4 n-1}$ in $\mathbb{R}^{4 n}$. Explicitly, this means that in distributional sense

$$
\partial_{\underline{X}_{0}} E_{0}(\underline{X})=\partial_{\underline{X}_{1}} E_{1}(\underline{X})=\partial_{\underline{X}_{2}} E_{2}(\underline{X})=\partial_{\underline{X}_{3}} E_{3}(\underline{X})=\delta(\underline{X})
$$

where moreover

$$
E_{r}(\underline{X})=\partial_{\underline{X}_{r}}\left[\frac{1}{4 n-2} \frac{1}{a_{4 n}} \frac{1}{|\underline{X}|^{4 n-2}}\right], \quad r=0,1,2,3,
$$

the expression between brackets being nothing but the fundamental solution of the (negative) Laplace operator $\left(-\Delta_{4 n}\right)$. Using (5) we thus also have that, for $r, s=0,1,2,3, r \neq s$,

$$
\partial_{\underline{X}_{r}} E_{s}(\underline{X})+\partial_{\underline{X}_{s}} E_{r}(\underline{X})=\left(\partial_{\underline{X}_{r}} \partial_{\underline{X}_{s}}+\partial_{\underline{X}_{s}} \partial_{\underline{X}_{r}}\right)\left[\frac{1}{4 n-2} \frac{1}{a_{4 n}} \frac{1}{|\underline{X}|^{4 n-2}}\right]=0
$$

in view of (4). We now introduce the Hermitean Cauchy kernels, given by

$$
\begin{aligned}
& \mathcal{E}_{0}=E_{0}-i E_{1}-j E_{2}-k E_{3}, \\
& \mathcal{E}_{1}=E_{0}-i E_{1}+j E_{2}+k E_{3}, \\
& \mathcal{E}_{2}=E_{0}+i E_{1}-j E_{2}+k E_{3}, \\
& \mathcal{E}_{3}=E_{0}+i E_{1}+j E_{2}-k E_{3},
\end{aligned}
$$

which may be rewritten as

$$
\mathcal{E}_{r}(\underline{Z})=\frac{1}{a_{4 n}} \frac{\underline{Z}_{r}^{\dagger}}{|\underline{Z}|^{4 n}}, \quad r=0,1,2,3 .
$$

Similarly to the complex Hermitean setting, see e.g. [8, 2], also here the Hermitean Cauchy kernels $\mathcal{E}_{0}, \mathcal{E}_{1}, \mathcal{E}_{2}$ and $\mathcal{E}_{3}$ are not the fundamental solutions of the respective Hermitean Dirac operators $\partial_{\underline{Z}_{0}}, \partial_{\underline{Z}_{1}}, \partial_{\underline{Z}_{2}}$ and $\partial_{\underline{Z}_{3}}$. However, the following proposition clarifies the role they are playing in the present context. 
Proposition 1. Introducing the circulant $(4 \times 4)$ matrices

$$
\mathcal{D}=\left(\begin{array}{cccc}
\partial_{Z_{0}} & \partial_{\underline{Z}_{3}} & \partial_{\underline{Z}_{2}} & \partial_{\underline{Z}_{1}} \\
\partial_{\underline{Z}_{1}} & \partial_{\underline{Z}_{0}} & \partial_{\underline{Z}_{3}} & \partial_{\underline{Z}_{2}} \\
\partial_{\underline{Z}} & \partial_{\underline{Z}_{1}} & \partial_{\underline{Z}_{0}} & \partial_{\underline{Z}} \\
\partial_{\underline{Z}_{3}} & \partial_{\underline{Z}_{2}} & \partial_{\underline{Z}_{1}} & \partial_{\underline{Z}_{0}}
\end{array}\right), \quad \mathcal{E}=\left(\begin{array}{cccc}
\mathcal{E}_{0} & \mathcal{E}_{3} & \mathcal{E}_{2} & \mathcal{E}_{1} \\
\mathcal{E}_{1} & \mathcal{E}_{0} & \mathcal{E}_{3} & \mathcal{E}_{2} \\
\mathcal{E}_{2} & \mathcal{E}_{1} & \mathcal{E}_{0} & \mathcal{E}_{3} \\
\mathcal{E}_{3} & \mathcal{E}_{2} & \mathcal{E}_{1} & \mathcal{E}_{0}
\end{array}\right)
$$

and

$$
\boldsymbol{\delta}=\left(\begin{array}{llll}
\delta & 0 & 0 & 0 \\
0 & \delta & 0 & 0 \\
0 & 0 & \delta & 0 \\
0 & 0 & 0 & \delta
\end{array}\right)
$$

one obtains that $\mathcal{D}^{T} \mathcal{E}=\boldsymbol{\delta}$.

Proof. First recall that circulant matrices form an algebra, whence it suffices to perform the calculations corresponding to the first column of $\mathcal{D}^{T} \mathcal{E}$, reading:

$$
\begin{aligned}
& \partial_{\underline{Z}_{0}} \mathcal{E}_{0}+\partial_{\underline{Z}_{1}} \mathcal{E}_{1}+\partial_{\underline{Z}_{2}} \mathcal{E}_{2}+\partial_{\underline{Z}_{3}} \mathcal{E}_{3}, \\
& \partial_{\underline{Z}_{3}} \mathcal{E}_{0}+\partial_{\underline{Z}_{0}} \mathcal{E}_{1}+\partial_{\underline{Z}_{1}} \mathcal{E}_{2}+\partial_{\underline{Z}_{2}} \mathcal{E}_{3}, \\
& \partial_{\underline{Z}_{2}} \mathcal{E}_{0}+\partial_{\underline{Z}_{3}} \mathcal{E}_{1}+\partial_{\underline{Z}_{0}} \mathcal{E}_{2}+\partial_{\underline{Z}_{1}} \mathcal{E}_{3}, \\
& \partial_{\underline{Z}_{1}} \mathcal{E}_{0}+\partial_{\underline{Z}_{2}} \mathcal{E}_{1}+\partial_{\underline{Z}_{3}} \mathcal{E}_{2}+\partial_{\underline{Z}_{0}} \mathcal{E}_{3} .
\end{aligned}
$$

For the first element we have:

$$
\begin{aligned}
& \frac{1}{16}\left[\left(\partial_{\underline{X}_{0}}+i \partial_{\underline{X}_{1}}+j \partial_{\underline{X}_{2}}+k \partial_{\underline{X}_{3}}\right)\left(E_{0}-i E_{1}-j E_{2}-k E_{3}\right)\right. \\
& +\left(\partial_{\underline{X}_{0}}+i \partial_{\underline{X}_{1}}-j \partial_{\underline{X}_{2}}-k \partial_{\underline{X}_{3}}\right)\left(E_{0}-i E_{1}+j E_{2}+k E_{3}\right) \\
& +\left(\underline{\partial}_{X_{0}}-i \partial_{X_{1}}+j \partial_{\underline{X}_{2}}-k \partial_{\underline{X}_{3}}\right)\left(E_{0}+i E_{1}-j E_{2}+k E_{3}\right) \\
& \left.+\left(\partial_{\underline{X}_{0}}-i \partial_{\underline{X}_{1}}-j \partial_{\underline{X}_{2}}+k \partial_{\underline{X}_{3}}\right)\left(E_{0}+i E_{1}+j E_{2}-k E_{3}\right)\right] \\
& =\frac{1}{16}\left[4 \delta\left(\underline{X}_{0}\right)+4 \delta\left(\underline{X}_{1}\right)+4 \delta\left(\underline{X}_{2}\right)+4 \delta\left(\underline{X}_{3}\right)\right]=\delta(\underline{Z}) .
\end{aligned}
$$

For the second one we have:

$$
\begin{gathered}
\frac{1}{16}\left[\left(\partial_{\underline{X}_{0}}-i \partial_{\underline{X}_{1}}-j \partial_{\underline{X}_{2}}+k \partial_{\underline{X}_{3}}\right)\left(E_{0}-i E_{1}-j E_{2}-k E_{3}\right)\right. \\
+\left(\partial_{\underline{X}_{0}}+i \partial_{\underline{X}_{1}}+j \partial_{\underline{X}_{2}}+k \partial_{\underline{X}_{3}}\right)\left(E_{0}-i E_{1}+j E_{2}+k E_{3}\right) \\
+\left(\underline{\partial}_{\underline{X}_{0}}+i \partial_{\underline{X}_{1}}-j \partial_{\underline{X}_{2}}-k{\underline{X_{3}}}_{3}\right)\left(E_{0}+i E_{1}-j E_{2}+k E_{3}\right) \\
\left.+\left(\partial_{\underline{X}_{0}}-i \partial_{\underline{X}_{1}}+j \partial_{\underline{X}_{2}}-k \partial_{\underline{X}_{3}}\right)\left(E_{0}+i E_{1}+j E_{2}-k E_{3}\right)\right] \\
=-\frac{j}{4}\left[\partial_{\underline{X}_{1}} E_{3}+\partial_{\underline{X}_{3}} E_{1}\right]=0
\end{gathered}
$$

which indeed vanishes seen (6). Similarly we see that $\partial_{\underline{Z}_{2}} \mathcal{E}_{0}+\partial_{\underline{Z}_{3}} \mathcal{E}_{1}+\partial_{\underline{Z}_{0}} \mathcal{E}_{2}+$ $\partial_{\underline{Z}_{1}} \mathcal{E}_{3}=0$ and $\partial_{\underline{Z}_{1}} \mathcal{E}_{0}+\partial_{\underline{Z}_{2}} \mathcal{E}_{1}+\partial_{\underline{Z}_{3}} \mathcal{E}_{2}+\partial_{\underline{Z}_{0}} \mathcal{E}_{3}=0$, which proves the statement. 
This result may be interpreted in the sense that the matrix $\mathcal{E}$ is a fundamental solution of the matricial Dirac operator $\mathcal{D}^{T}$. It is exactly this observation which will lead to the construction of a matrix quaternionic Hermitean Cauchy integral formula. But first, inspired by this proposition, we will introduce an auxiliary definition, as well as some more notations.

Let $\Omega$ be an open set in $\mathbb{R}^{4 n}$, and consider functions $g_{0}, g_{1}, g_{2}$ and $g_{3}$, which are continuously differentiable in $\Omega \subset \mathbb{R}^{4 n}$ and take their values in $\mathbb{H}_{4 n}$. Similarly to the structure of the matrix $\mathcal{E}$, we then introduce the $(4 \times 4)$ circulant matrix function

$$
\boldsymbol{G}=\left(\begin{array}{cccc}
g_{0} & g_{3} & g_{2} & g_{1} \\
g_{1} & g_{0} & g_{3} & g_{2} \\
g_{2} & g_{1} & g_{0} & g_{3} \\
g_{3} & g_{2} & g_{1} & g_{0}
\end{array}\right)
$$

Definition 3. The matrix function $\boldsymbol{G}$ is called (left) $\mathbf{Q}$-Hermitean monogenic in $\Omega$ if and only if

$$
\mathcal{D}^{T} \boldsymbol{G}=\boldsymbol{O} \text { in } \Omega
$$

where $\boldsymbol{O}$ denotes the matrix with zero entries.

Explicitly, the above system (8) reads

$$
\begin{aligned}
& \partial_{\underline{Z}_{0}} g_{0}+\partial_{\underline{Z}_{1}} g_{1}+\partial_{\underline{Z}_{2}} g_{2}+\partial_{\underline{Z}_{3}} g_{3}=0 \\
& \partial_{\underline{Z}_{3}} g_{0}+\partial_{\underline{Z}_{0}} g_{1}+\partial_{\underline{Z}_{1}} g_{2}+\partial_{\underline{Z}_{2}} g_{3}=0 \\
& \partial_{\underline{Z}_{2}} g_{0}+\partial_{\underline{Z}_{3}} g_{1}+\partial_{\underline{Z}_{0}} g_{2}+\partial_{\underline{Z}_{1}} g_{3}=0 \\
& \partial_{\underline{Z}_{1}} g_{0}+\partial_{\underline{Z}_{2}} g_{1}+\partial_{\underline{Z}_{3}} g_{2}+\partial_{\underline{Z}_{0}} g_{3}=0
\end{aligned}
$$

It thus is directly clear that the Q-Hermitean monogenicity of $\boldsymbol{G}$ will, in general, not imply the $q$-Hermitean monogenicity of its components. There is, however, an important exception. Indeed, when considering the matrix function

$$
\boldsymbol{G}_{0}=\left(\begin{array}{cccc}
g & 0 & 0 & 0 \\
0 & g & 0 & 0 \\
0 & 0 & g & 0 \\
0 & 0 & 0 & g
\end{array}\right)
$$

it directly follows that $\boldsymbol{G}_{0}$ is $\mathbf{Q}$-Hermitean monogenic if and only if the function $g$ is $q$-Hermitean monogenic. Moreover, it is worth observing that the quaternionic Dirac matrix $\mathcal{D}$ in some sense factorizes the Laplacian, since

$$
16\left(\mathcal{D}^{T} \mathcal{D}^{\dagger}\right)=\left(\begin{array}{cccc}
\Delta_{4 n} & 0 & 0 & 0 \\
0 & \Delta_{4 n} & 0 & 0 \\
0 & 0 & \Delta_{4 n} & 0 \\
0 & 0 & 0 & \Delta_{4 n}
\end{array}\right)
$$

where the notation $\mathcal{D}^{\dagger}$ means that $\mathbb{H}$-conjugation is applied to all entries of $\mathcal{D}$. 


\section{Cauchy integral formulae}

In [17], while attempting to obtain a Martinelli-Bochner type formula in the several quaternionic variables setting, the authors proposed a generalized Cauchy-integral formula for the operator $\partial_{\underline{Z}_{0}}$, by making essential use of the kernel $\mathcal{E}_{0}$, which, however, fails to be $q$-Hermitean monogenic. Now Proposition 1 suggests that, in order to establish a more suitable Cauchy formula in the quaternionic Hermitean context, the functions $\mathcal{E}_{0}, \mathcal{E}_{1}, \mathcal{E}_{2}$ and $\mathcal{E}_{3}$ need to be involved together.

To this end, let $\Gamma$ be a bounded and simply connected domain in $\mathbb{R}^{4 n}$ with smooth boundary $\partial \Gamma$. Then we introduce the unit normal vector $\underline{n}(\underline{X}) \equiv \underline{n}_{0}(\underline{X})$ on $\partial \Gamma$ at a point $\underline{X} \in \partial \Gamma$, as well as its "twisted" versions $\underline{n}_{1}(\underline{X}), \underline{n}_{2}(\underline{X})$ and $\underline{n}_{3}(\underline{X})$. As has been done above for the vector variables and the corresponding Dirac operators, we may consider its quaternionic Hermitean counterparts

$$
\begin{aligned}
\mathcal{N}_{0} & =\frac{1}{16}\left(\underline{n}_{0}+i \underline{n}_{1}+j \underline{n}_{2}+k \underline{n}_{3}\right), \\
\mathcal{N}_{1} & =\frac{1}{16}\left(\underline{n}_{0}+i \underline{n}_{1}-j \underline{n}_{2}-k \underline{n}_{3}\right), \\
\mathcal{N}_{2} & =\frac{1}{16}\left(\underline{n}_{0}-i \underline{n}_{1}+j \underline{n}_{2}-k \underline{n}_{3}\right), \\
\mathcal{N}_{3} & =\frac{1}{16}\left(\underline{n}_{0}-i \underline{n}_{1}-j \underline{n}_{2}+k \underline{n}_{3}\right),
\end{aligned}
$$

where however, an additional multiplicative constant has been introduced deliberately. With $\mathcal{N}_{0}, \mathcal{N}_{1}, \mathcal{N}_{2}, \mathcal{N}_{3}$, we then associate in the usual way the circulant matrix $\mathcal{N}$, which allows us to formulate the following result.

Theorem 1. Let $\boldsymbol{F}$ and $\boldsymbol{G}$ be circulant matrix functions of the form (7), with entries in $C^{1}\left(\Omega, \mathbb{H}_{4 n}\right)$, and let $\Gamma \subset \Omega$ be a $4 n$-dimensional compact differentiable and oriented manifold with $C^{\infty}{ }_{-s m o o t h}$ boundary $\partial \Gamma$. It then holds that

$$
\int_{\Gamma}\left[\left(\boldsymbol{F} \mathcal{D}^{T}\right) \boldsymbol{G}+\boldsymbol{F}\left(\mathcal{D}^{T} \boldsymbol{G}\right)\right] d V=\int_{\partial \Gamma} \boldsymbol{F} \mathcal{N}^{T} \boldsymbol{G} d S,
$$

where $d V$ and $d S$ stand for the elementary volume and scalar surface measures, respectively.

Proof. The above result is nothing but a reformulation in matricial form of the Hermitean Clifford-Stokes theorems established in [17].

From now on $\Gamma^{+}$will stand for the interior of $\Gamma$ and $\Gamma^{-}$for $\Omega \backslash \Gamma$. We use the notations $\underline{Y}=\underline{Y}_{0}$ for the Clifford vector associated to points in $\Gamma^{ \pm}$. Its quaternionic Hermitean counterpart is denoted by $\underline{V}=\underline{V}_{0}$.

On account of Theorem 1 we then arrive at a $\mathbf{Q}$-Hermitean version of the Borel-Pompeiu formula. 
Theorem 2 (Q-Hermitean Borel-Pompeiu formula). Let $\boldsymbol{G}$ be a circulant matrix function of the form (7) with entries in $C^{1}\left(\Omega, \mathbb{H}_{4 n}\right)$, and let as above $\Gamma \subset \Omega$ be a 4 -dimensional compact differentiable and oriented manifold with $C^{\infty}{ }_{- \text {smooth }}$ boundary $\partial \Gamma$. It then holds that

$$
\begin{gathered}
\int_{\partial \Gamma} \mathcal{E}(\underline{Z}-\underline{V}) \mathcal{N}^{T}(\underline{Z}) \boldsymbol{G}(\underline{X}) d S(\underline{X})-\int_{\Gamma} \mathcal{E}(\underline{Z}-\underline{V})\left[\mathcal{D}^{T} \boldsymbol{G}(\underline{X})\right] d V(\underline{X}) \\
= \begin{cases}\boldsymbol{G}(\underline{Y}), & \underline{Y} \in \Gamma^{+} ; \\
\boldsymbol{O}, & \underline{Y} \in \Gamma^{-} .\end{cases}
\end{gathered}
$$

Proof. First, let $\underline{Y} \in \Gamma^{-}$. Then the matrix function $\mathcal{E}(\underline{Z}-\underline{V})$ is continuously differentiable in $\Gamma^{+}$, so that the quaternionic Hermitean Clifford-Stokes Theorem 1 can be applied, yielding the desired statement, since we have that in $\Omega$

$$
\mathcal{D}^{T} \mathcal{E}=\mathcal{E} \mathcal{D}^{T}=\boldsymbol{O} .
$$

Next, let $\underline{Y} \in \Gamma^{+}$. Take $R>0$ such that $B(\underline{Y}, R) \subset \Gamma^{+}$. Invoking the previous case, we may then write

$$
\begin{aligned}
\int_{\partial(\Gamma \backslash B(\underline{Y}, R))} & \mathcal{E}(\underline{Z}-\underline{V}) \mathcal{N}^{T}(\underline{Z}) \boldsymbol{G}(\underline{X}) d S(\underline{X}) \\
& -\int_{\Gamma \backslash B(\underline{Y}, R)} \mathcal{E}(\underline{Z}-\underline{V})\left[\mathcal{D}^{T} \boldsymbol{G}(\underline{X})\right] d V(\underline{X})=\boldsymbol{O} .
\end{aligned}
$$

Taking limits for $R \rightarrow 0+$ the second term at the left-hand side yields

$$
\lim _{R \rightarrow 0} \int_{\Gamma \backslash B(\underline{Y}, R)} \mathcal{E}(\underline{Z}-\underline{V})\left[\mathcal{D}^{T} \boldsymbol{G}(\underline{X})\right] d V(\underline{X})=\int_{\Gamma} \mathcal{E}(\underline{Z}-\underline{V})\left[\mathcal{D}^{T} \boldsymbol{G}(\underline{X})\right] d V(\underline{X}),
$$

since the integrand only contains functions which are integrable on $\Gamma$. For the integral over the boundary we have

$$
\begin{aligned}
& \int_{\partial(\Gamma \backslash B(\underline{Y}, R))} \mathcal{E}(\underline{Z}-\underline{V}) \mathcal{N}^{T}(\underline{Z}) \boldsymbol{G}(\underline{X}) d S(\underline{X}) \\
& \quad=\int_{\partial \Gamma} \mathcal{E}(\underline{Z}-\underline{V}) \mathcal{N}^{T}(\underline{Z}) \boldsymbol{G}(\underline{X}) d S(\underline{X})-\int_{\partial B(\underline{Y}, R)} \mathcal{E}(\underline{Z}-\underline{V}) \mathcal{N}^{T}(\underline{Z}) \boldsymbol{G}(\underline{X}) d S(\underline{X}) .
\end{aligned}
$$

In order to calculate the last integral in the above expression, we note that it equals

$\int_{\partial B(\underline{Y}, R)} \mathcal{E}(\underline{Z}-\underline{V}) \mathcal{N}^{T}(\underline{Z}) \boldsymbol{G}(\underline{Y}) d S(\underline{X})+\int_{\partial B(\underline{Y}, R)} \mathcal{E}(\underline{Z}-\underline{V}) \mathcal{N}^{T}(\underline{Z})(\boldsymbol{G}(\underline{X})-\boldsymbol{G}(\underline{Y})) d S(\underline{X})$.

By direct calculation of the first term we obtain

$$
\lim _{R \rightarrow 0} \int_{\partial B(\underline{Y}, R)} \mathcal{E}(\underline{Z}-\underline{V}) \mathcal{N}^{T}(\underline{Z}) \boldsymbol{G}(\underline{Y}) d S(\underline{X})=\boldsymbol{G}(\underline{Y}),
$$

while the second term may be shown to converge to $\boldsymbol{O}$ for $R \rightarrow 0+$, which concludes the proof. 
Theorem 2 now leads to the following Hermitean Cauchy integral formulae for Q-Hermitean monogenic matrix functions and $q$-Hermitean monogenic functions, respectively.

Theorem 3 (Q-Hermitean Cauchy integral formula). If the matrix function $\boldsymbol{G}$, (7), is $\mathbf{Q}$-Hermitean monogenic in $\Omega$ then

$$
\int_{\partial \Gamma} \mathcal{E}(\underline{Z}-\underline{V}) \mathcal{N}^{T}(\underline{Z}) \boldsymbol{G}(\underline{X}) d S(\underline{X})=\left\{\begin{array}{ll}
\boldsymbol{G}(\underline{Y}), & \underline{Y} \in \Gamma^{+} \\
\boldsymbol{O}, & \underline{Y} \in \Gamma^{-}
\end{array} .\right.
$$

Proof. Seen the Q-Hermitean monogenicity of the matrix function $\boldsymbol{G}$, the integral over the domain $\Gamma$ in the result of Theorem 2 vanishes and the Cauchy integral formula follows.

Theorem 4 ( $q$-Hermitean Cauchy integral formula). If the function g is q-Hermitean monogenic in $\Omega$ then

$$
\int_{\partial \Gamma} \mathcal{E}(\underline{Z}-\underline{V}) \mathcal{N}^{T}(\underline{Z}) \boldsymbol{G}_{0}(\underline{X}) d S(\underline{X})= \begin{cases}\boldsymbol{G}_{0}(\underline{Y}), & \underline{Y} \in \Gamma^{+} \\ \boldsymbol{O}, & \underline{Y} \in \Gamma^{-}\end{cases}
$$

where $\boldsymbol{G}_{0}$ is the corresponding matrix (9).

Proof. The assumed $q$-Hermitean monogenicity of the function $g$ entails the QHermitean monogenicity of the matrix function $\boldsymbol{G}_{0}$; the result then follows from Theorem 3 .

Theorem 4 may be considered as a Hermitean Cauchy integral formula for the $q$-Hermitean monogenic function $g$; therefore the matrix $\mathcal{E}$ appearing in this formula is called de quaternionic Hermitean Cauchy kernel.

\section{A Martinelli-Bochner type formula}

The aim of this section is to recover from our matricial integral representation formulae the Martinelli-Bochner type formula established in [17] as a special case. To this end we will take in the $\mathbf{Q}$-Hermitean Borel-Pompeiu formula of Theorem 2 a matrix function of the form $\boldsymbol{G}_{0},(9)$. Comparing the entries of the corresponding matrices at both sides of the equality leads, after some direct calculations, to four expressions, the first one of which reads

$$
\sum_{s=0}^{3}\left[\int_{\partial \Gamma} \mathcal{E}_{s}(\underline{Z}-\underline{V}) \mathcal{N}_{s}(\underline{Z}) g(\underline{X}) d S(\underline{X})-\int_{\Gamma} \mathcal{E}_{s}(\underline{Z}-\underline{V})\left(\partial_{\underline{Z}_{s}} g(\underline{X})\right) d V(\underline{X})\right]=g(\underline{Y}) .
$$

Let $B(\underline{Y}, R)$ be a ball which is contained in $\Gamma$. We then have

$$
\lim _{R \rightarrow 0} \int_{\Gamma \backslash B(\underline{Y}, R)} \mathcal{E}_{s}(\underline{Z}-\underline{V})\left[\partial_{\underline{Z}_{s}} g(\underline{X})\right] d V(\underline{X})=\int_{\Gamma} \mathcal{E}_{s}(\underline{Z}-\underline{V})\left[\partial_{\underline{Z}_{s}} g(\underline{X})\right] d V(\underline{X}),
$$


since the integrand only contains functions which are integrable on $\Gamma$, whence some rearrangements in (10) give

$$
\begin{aligned}
\int_{\partial \Gamma} \mathcal{E}_{0}(\underline{Z}-\underline{V}) \mathcal{N}_{0}(\underline{Z}) g(\underline{X}) d S(\underline{X})-\int_{\Gamma} \mathcal{E}_{0}(\underline{Z}-\underline{V})\left[\underline{\partial}_{\underline{Z}_{0}} g(\underline{X})\right] d V(\underline{X}) \\
+\lim _{R \rightarrow 0} \sum_{s=1}^{3}\left[\int_{\partial(\Gamma \backslash B(\underline{Y}, R))} \mathcal{E}_{s}(\underline{Z}-\underline{V}) \mathcal{N}_{s}(\underline{Z}) g(\underline{X}) d S(\underline{X})\right. \\
\left.-\int_{\Gamma \backslash B(\underline{Y}, R)} \mathcal{E}_{s}(\underline{Z}-\underline{V})\left[\partial_{\underline{Z}_{s}} g(\underline{X})\right] d V(\underline{X})\right] \\
+\lim _{R \rightarrow 0} \sum_{s=1}^{3} \int_{\partial B(\underline{Y}, R)} \mathcal{E}_{s}(\underline{Z}-\underline{V}) \mathcal{N}_{s}(\underline{Z}) g(\underline{X}) d S(\underline{X})=g(\underline{Y}) .
\end{aligned}
$$

Applying the quaternionic Hermitean Clifford-Stokes formula to the second sum at the left hand side of the above equality leads to

$$
\begin{aligned}
\int_{\partial \Gamma} \mathcal{E}_{0}(\underline{Z}-\underline{V}) \mathcal{N}_{0}(\underline{Z}) g(\underline{X}) d S(\underline{X})-\int_{\Gamma} \mathcal{E}_{0}(\underline{Z}-\underline{V})\left[\partial_{\underline{Z}_{0}} g(\underline{X})\right] d V(\underline{X}) \\
+\lim _{R \rightarrow 0} \sum_{s=1}^{3} \int_{\Gamma \backslash B(\underline{Y}, R)}\left[\mathcal{E}_{s}(\underline{Z}-\underline{V}) \partial_{\underline{Z}_{s}}\right] g(\underline{X}) d V(\underline{X}) \\
+\lim _{R \rightarrow 0} \sum_{s=1}^{3} \int_{\partial B(\underline{Y}, R)} \mathcal{E}_{s}(\underline{Z}-\underline{V}) \mathcal{N}_{s}(\underline{Z}) g(\underline{X}) d S(\underline{X})=g(\underline{Y}) .
\end{aligned}
$$

Next we use the identity

$$
\sum_{s=0}^{3}\left[\mathcal{E}_{s}(\underline{Z}-\underline{V}) \partial_{\underline{Z}_{s}}\right]=0, \quad \text { for } \underline{Z} \neq \underline{V}
$$

in order to arrive at

$$
\begin{aligned}
\int_{\partial \Gamma} \mathcal{E}_{0}(\underline{Z}-\underline{V}) \mathcal{N}_{0}(\underline{Z}) g(\underline{X}) d S(\underline{X})-\int_{\Gamma} \mathcal{E}_{0}(\underline{Z}-\underline{V})\left[\partial_{\underline{Z}_{0}} g(\underline{X})\right] d V(\underline{X}) \\
-\lim _{R \rightarrow 0} \int_{\Gamma \backslash B(\underline{Y}, R)}\left[\mathcal{E}_{0}(\underline{Z}-\underline{V}) \partial_{\underline{Z}_{0}}\right] g(\underline{X}) d V(\underline{X}) \\
+\lim _{R \rightarrow 0} \sum_{s=1}^{3} \int_{\partial B(\underline{Y}, R)} \mathcal{E}_{s}(\underline{Z}-\underline{V}) \mathcal{N}_{s}(\underline{Z}) g(\underline{X}) d S(\underline{X})=g(\underline{Y}) .
\end{aligned}
$$

Direct calculations show that

$$
\lim _{R \rightarrow 0} \sum_{s=1}^{3} \int_{\partial B(\underline{Y}, R)} \mathcal{E}_{s}(\underline{Z}-\underline{V}) \mathcal{N}_{s}(\underline{Z}) g(\underline{X}) d S(\underline{X})=\frac{3}{4} g(\underline{Y}),
$$


and hence

$$
\begin{gathered}
\int_{\partial \Gamma} \mathcal{E}_{0}(\underline{Z}-\underline{V}) \mathcal{N}_{0}(\underline{Z}) g(\underline{X}) d S(\underline{X})-\int_{\Gamma} \mathcal{E}_{0}(\underline{Z}-\underline{V})\left[\partial_{\underline{Z}_{0}} g(\underline{X})\right] d V(\underline{X}) \\
-\lim _{R \rightarrow 0} \int_{\Gamma \backslash B(\underline{Y}, R)}\left[\mathcal{E}_{0}(\underline{Z}-\underline{V}) \partial_{\underline{Z}_{0}} g(\underline{X}) d V(\underline{X})\right]=\frac{1}{4} g(\underline{Y}) .
\end{gathered}
$$

Theorem 4.3 in [17] follows from the above formula after taking into account the language-translations $\mathcal{E}_{0} \mathcal{N}_{0}=\frac{1}{4} E_{\underline{q}} n_{\underline{q}}$ and $\mathcal{E}_{0} \partial_{\underline{Z}_{0}}=\frac{1}{4} E_{\underline{q}} \partial_{\underline{q}}$.

Remark 1. The three remaining identities which follow from taking $\boldsymbol{G}=\boldsymbol{G}_{0}$ in the Q-Hermitean Borel-Pompeiu formula, have the form

$$
\begin{aligned}
& \int_{\partial \Gamma}\left[\mathcal{E}_{1}(\underline{Z}-\underline{V}) \mathcal{N}_{0}(\underline{Z})+\mathcal{E}_{0}(\underline{Z}-\underline{V}) \mathcal{N}_{3}(\underline{Z})\right. \\
& \left.+\mathcal{E}_{3}(\underline{Z}-\underline{V}) \mathcal{N}_{2}(\underline{Z})+\mathcal{E}_{2}(\underline{Z}-\underline{V}) \mathcal{N}_{1}(\underline{Z})\right] g(\underline{X}) d S(\underline{X}) \\
& =\int_{\Gamma}\left[\mathcal{E}_{1}(\underline{Z}-\underline{V})\left(\partial_{\underline{Z}_{0}} g(\underline{X})\right)+\mathcal{E}_{0}(\underline{Z}-\underline{V})\left(\partial_{\underline{Z}_{3}} g(\underline{X})\right)\right. \\
& \left.+\mathcal{E}_{3}(\underline{Z}-\underline{V})\left(\partial_{\underline{Z}_{2}} g(\underline{X})\right)+\mathcal{E}_{2}(\underline{Z}-\underline{V})\left(\partial_{\underline{Z}_{1}} g(\underline{X})\right)\right] d V(\underline{X}), \\
& \int_{\partial \Gamma}\left[\mathcal{E}_{2}(\underline{Z}-\underline{V}) \mathcal{N}_{0}(\underline{Z})+\mathcal{E}_{1}(\underline{Z}-\underline{V}) \mathcal{N}_{3}(\underline{Z})\right. \\
& \left.+\mathcal{E}_{0}(\underline{Z}-\underline{V}) \mathcal{N}_{2}(\underline{Z})+\mathcal{E}_{3}(\underline{Z}-\underline{V}) \mathcal{N}_{1}(\underline{Z})\right] g(\underline{X}) d S(\underline{X}) \\
& =\int_{\Gamma}\left[\mathcal{E}_{2}(\underline{Z}-\underline{V})\left(\partial_{\underline{Z}_{0}} g(\underline{X})\right)+\mathcal{E}_{1}(\underline{Z}-\underline{V})\left(\partial_{\underline{Z}_{3}} g(\underline{X})\right)\right. \\
& \left.+\mathcal{E}_{0}(\underline{Z}-\underline{V})\left(\partial_{\underline{Z}_{2}} g(\underline{X})\right)+\mathcal{E}_{3}(\underline{Z}-\underline{V})\left(\partial_{\underline{Z}_{1}} g(\underline{X})\right)\right] d V(\underline{X}), \\
& \int_{\partial \Gamma}\left[\mathcal{E}_{3}(\underline{Z}-\underline{V}) \mathcal{N}_{0}(\underline{Z})+\mathcal{E}_{2}(\underline{Z}-\underline{V}) \mathcal{N}_{3}(\underline{Z})\right. \\
& \left.+\mathcal{E}_{1}(\underline{Z}-\underline{V}) \mathcal{N}_{2}(\underline{Z})+\mathcal{E}_{0}(\underline{Z}-\underline{V}) \mathcal{N}_{1}(\underline{Z})\right] g(\underline{X}) d S(\underline{X}) \\
& =\int_{\Gamma}\left[\mathcal{E}_{3}(\underline{Z}-\underline{V})\left(\partial_{\underline{Z}_{0}} g(\underline{X})\right)+\mathcal{E}_{2}(\underline{Z}-\underline{V})\left(\partial_{\underline{Z}_{3}} g(\underline{X})\right)\right. \\
& \left.+\mathcal{E}_{1}(\underline{Z}-\underline{V})\left(\partial_{\underline{Z}_{2}} g(\underline{X})\right)+\mathcal{E}_{0}(\underline{Z}-\underline{V})\left(\partial_{\underline{Z}_{1}} g(\underline{X})\right)\right] d V(\underline{X}) .
\end{aligned}
$$

Although these identities are a direct consequences of the theorem, they may-of course-also be proven directly by means of Stokes's formula.

\section{Conclusions}

In this paper we have established a true Cauchy integral representation formula for $q$-Hermitean monogenic functions. At the same time, we have laid the foundations 
for a circulant $(4 \times 4)$ matrix function theory centered around the null solutions of a quaternionic matrix Hermitean Dirac operator, which, in origin, shows conceptual similarities to the circulant $(2 \times 2)$ matrix Hermitean Clifford analysis mentioned before. The results obtained constitute important building blocks for the further development of this theory and for the treatment of the corresponding boundary value problems. This will be the subject of forthcoming papers.

\section{References}

[1] R. Abreu Blaya et al., Hermitean Cauchy integral decomposition of continuous functions on hypersurfaces, Bound. Value Probl. vol.2008 (2008), article ID 425256, 16 pages.

[2] R. Abreu Blaya et al., A Hermitean Cauchy formula on a domain with fractal boundary, J. Math. Anal. Appl. 369 (2010), 273-282.

[3] R. Abreu Blaya, J. Bory Reyes, T. Moreno García, Hermitian decomposition of continuous functions on a fractal surface, Bull. Braz. Math. Soc. 40(1) (2009), 107-115.

[4] F. Brackx, R. Delanghe, F. Sommen, Clifford Analysis, Research Notes in Mathematics 76, Pitman, Boston, MA, 1982.

[5] F. Brackx et al., Fundaments of Hermitean Clifford analysis - Part I: Complex structure, Complex Anal. Oper. Theory 1(3) (2007), 341-365.

[6] F. Brackx et al., Fundaments of Hermitean Clifford analysis - Part II: Splitting of h-monogenic equations, Complex Var. Elliptic Equ. 52(10-11) (2007), 1063-1079.

[7] F. Brackx, H. De Schepper, F. Sommen, The Hermitian Clifford analysis toolbox, Adv. Appl. Clifford Alg. 18(3-4) (2008), 451-487.

[8] F. Brackx, B. De Knock, H. De Schepper, F. Sommen, On Cauchy and Martinelli-Bochner integral formulae in Hermitean Clifford analysis, Bull. Braz. Math. Soc. 40(3) (2009), 395-416.

[9] A. Damiano, D. Eelbode, I. Sabadini, Quaternionic Hermitian spinor systems and compatibility conditions, Adv. Geom. 11 (2011), 169-189.

[10] A. Damiano, D. Eelbode, I. Sabadini, Algebraic analysis of Hermitian monogenic functions, C.R. Acad. Sci. Paris Ser.I 346 (2008), 139-142.

[11] R. Delanghe, F. Sommen, V. Souček, Clifford Algebra and Spinor-Valued Functions, Kluwer Academic Publishers, Dordrecht, 1992. 
[12] K. Gürlebeck, K. Habetha, W. Sprössig, Holomorphic functions in the plane and $n$-dimensional space, translated from the 2006 German original, Birkhäuser Verlag, Basel, 2008.

[13] K. Gürlebeck, W. Sprössig, Quaternionic and Clifford Calculus for Physicists and Engineers, Wiley, Chichester, 1998.

[14] J. Gilbert, M. Murray, Clifford Algebras and Dirac Operators in Harmonic Analysis, Cambridge University Press, Cambridge, 1991.

[15] S. Krantz, Function theory of several complex variables, 2nd edition, Wadsworth \& Brooks/Cole, Pacific Grove, 1992.

[16] A. Kytmanov, The Bochner-Martinelli integral and its applications, Birkhaüser, Basel-Boston-Berlin, 1995.

[17] D. Peña-Peña, I. Sabadini, F. Sommen, Quaternionic Clifford analysis: the Hermitian setting, Complex Anal. Oper. Theory 1 (2007), 97-113.

[18] R. Rocha-Chávez, M. Shapiro, F. Sommen, Integral theorems for functions and differential forms in $\mathbb{C}^{m}$, Research Notes in Mathematics 428, Chapman \& Hall/CRC, Boca Raton, FL, 2002.

[19] J. Ryan, Complexified Clifford analysis, Comp. Var. Theory Applic. 1(1) (1982/83), 119-149.

[20] I. Sabadini, F. Sommen, Hermitian Clifford analysis and resolutions, Math. Methods Appl. Sci. 25(16-18) (2002), 1395-1413.

R. Abreu Blaya:

Facultad de Informática y Matemática, Universidad de Holguín, Holguín 80100, Cuba. (E-mail: rabreu@facinf.uho.edu.cu)

J. Bory Reyes:

Departamento de Matemática, Universidad de Oriente, Santiago de Cuba 90500,

Cuba. (E-mail: jbory@rect.uo.edu.cu)

F. Brackx:

Department of Mathematical Analysis, Faculty of Engineering, Ghent University, Galglaan 2, 9000 Gent, Belgium. (E-mail: fb@cage.UGent.be)

H. De Schepper:

Department of Mathematical Analysis, Faculty of Engineering, Ghent University, Galglaan 2, 9000 Gent, Belgium. (E-mail: hds@cage.UGent.be)

F. Sommen:

Department of Mathematical Analysis, Faculty of Engineering, Ghent University, Galglaan 2, 9000 Gent, Belgium. (E-mail: fs@cage.UGent.be) 